Case Report

\title{
Laparoscopic Resection of Recurrence from Hepatocellular Carcinoma after Liver Transplantation: Case Reports and Review of the Literature
}

\author{
Mushegh A. Sahakyan, ${ }^{1,2,3}$ Airazat M. Kazaryan, ${ }^{1,4}$ Ewa Pomianowska, \\ Andreas Abildgaard, ${ }^{6}$ Pål-Dag Line, ${ }^{7}$ Bjørn Atle Bjørnbeth, ${ }^{5}$ Bjørn Edwin, ${ }^{1,2,5}$ \\ and Bård Ingvald Røsok ${ }^{5}$
}

${ }^{1}$ The Intervention Centre, Oslo University Hospital, Rikshospitalet, 0027 Oslo, Norway

${ }^{2}$ Institute of Clinical Research, Medical Faculty, University of Oslo, 0318 Oslo, Norway

${ }^{3}$ Department of Surgery No. 1, Yerevan State Medical University after M. Heratsi, 0025 Yerevan, Armenia

${ }^{4}$ Department of Gastrointestinal Surgery, Akershus University Hospital, 1478 Lørenskog, Norway

${ }^{5}$ Department of HPB Surgery, Oslo University Hospital, Rikshospitalet, 0407 Oslo, Norway

${ }^{6}$ Department of Radiology, Oslo University Hospital, Rikshospitalet, 0027 Oslo, Norway

${ }^{7}$ Department of Transplantation Medicine, Oslo University Hospital, Rikshospitalet, 0424 Oslo, Norway

Correspondence should be addressed to Mushegh A. Sahakyan; sahakyan.mushegh@gmail.com

Received 12 November 2015; Revised 29 January 2016; Accepted 15 February 2016

Academic Editor: Constantine Gennatas

Copyright (C) 2016 Mushegh A. Sahakyan et al. This is an open access article distributed under the Creative Commons Attribution License, which permits unrestricted use, distribution, and reproduction in any medium, provided the original work is properly cited.

\begin{abstract}
Background. Recurrence of hepatocellular carcinoma (HCC) after liver transplantation (LT) indicates a poor prognosis. Surgery is considered the only curative option for selected patients with HCC recurrence following LT. Traditionally, the preference is given to the open approach. Methods. In this report, we present two cases of laparoscopic resections (LR) for recurrent HCC after LT, performed at Oslo University Hospital, Rikshospitalet. Results. Both procedures were executed without intraoperative and postoperative adverse events. Whereas one of the patients had a recurrence one year after LR, the other patient did not have any sign of disease during 3-year follow-up. Conclusions. We argue that, in selected cases, patients with HCC recurrence following LT may benefit from LR due to its limited tissue trauma and timely start of subsequent treatment if curative resection cannot be obtained. In patients with relatively favorable prognosis, LR facilitates postoperative recovery course and avoids unnecessary laparotomy.
\end{abstract}

\section{Introduction}

Hepatocellular carcinoma (HCC) is the fifth most common cancer and the second leading cause of cancer related death in the world with more than a half million new cases annually $[1,2]$.

The key aspect in successful treatment of HCC is linked to proper preoperative staging. The most widely adopted staging system is the Barcelona Clinic Liver Cancer (BCLC) system, based on tumor stage, severity of liver disease, and performance status of the patients to different therapeutic alternatives. According to BCLC recommendations, the patients with early HCC are suitable candidates for radical treatment (i.e., resection, liver transplantation, or radio frequency ablation). Among these approaches, liver transplantation (LT) seems to be an attractive therapeutic option, particularly in patients with significant liver parenchymal disease (ChildPugh B and C), as it has the potential to cure both the tumor and underlying liver disease [3].

However LT is often limited to early stages of disease in order to ensure outcomes that are comparable to those of liver transplantation for noncancer related indications. Selection criteria, such as Milan (a single tumor $\leq 5 \mathrm{~cm}$ or $\leq 3$ tumors $\leq 3 \mathrm{~cm}$ without signs of macrovascular invasion), UCSF (a single tumor $\leq 6.5 \mathrm{~cm}$, or $\leq 3$ tumors with the largest being $\leq 4.5 \mathrm{~cm}$, and a total tumor burden of $\leq 8 \mathrm{~cm}$ ), and up-to-seven 

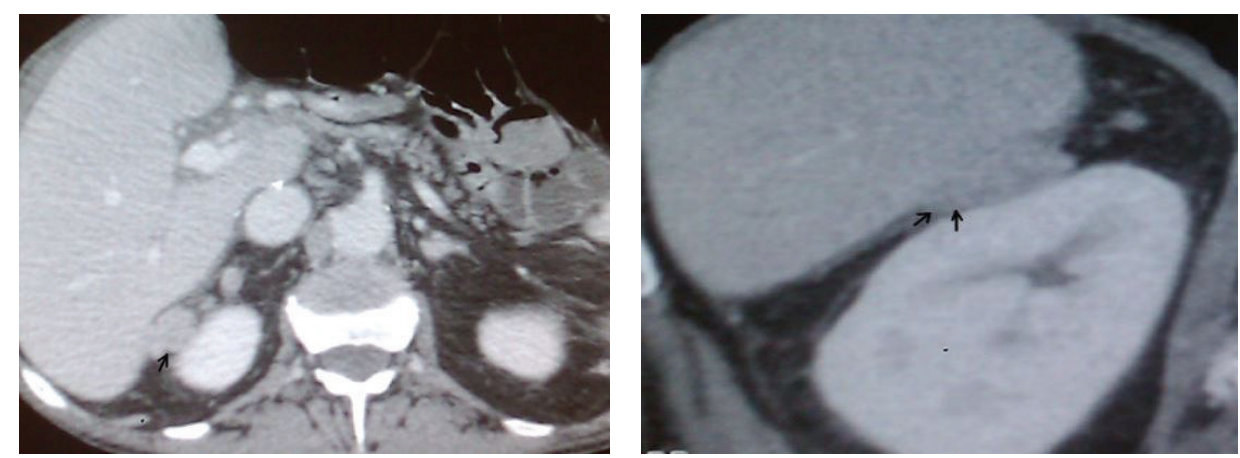

FIGURE 1: CT with intravenous contrast. Extrahepatic lesion between liver segment 6 and right kidney, largest diameter $2 \mathrm{~cm}$.

(the sum of the number of nodules and of the diameter of the largest tumor (in $\mathrm{cm}$ ) not exceeding seven, in absence of macrovascular invasion and extrahepatic spread), have been introduced up to date [4-8].

At the same time, recurrence of HCC after LT still occurs and is to a large extent related to tumor stage. Relapse after transplantation is usually associated with poor prognosis, since in most cases it must be considered as a sign of systemic disease. Extrahepatic lesions, present in majority of these patients, are not amenable to curative treatment, thereby being considered for palliative therapy only. However, the efficacy of the latter remains unsatisfactory [9]. Several authors emphasized the curative role of surgery for HCC recurrences, indicating that longer time interval between LT and the recurrence and feasibility of resection are associated with improved survival [10-12].

Today, laparoscopic surgery is comparable to open technique for primary resection in a large fraction of patients with HCC [13]. However, in terms of tumor recurrences, a conventional open technique is usually preferred, perhaps considering the fact that patients had previously undergone major surgical procedure. In our review of current literature, we are not aware of any report concerning laparoscopic approach for HCC recurrence after LT. Herein we report on two cases of recurrences following LT that were treated by laparoscopic resections at our institution.

\section{Materials and Methods}

Hospital records review revealed that 89 patients with HCC on final pathology underwent LT at Oslo University Hospital, Rikshospitalet, from 2004 to 2014. Eighteen of these patients (20.2\%) had recurrence during follow-up. Of these, two with locoregional isolated recurrences and performance status of 0 according to Eastern Cooperative Oncology Group (ECOG) scale were considered eligible for curative resection. Laparoscopic approach was applied.

\section{Results}

3.1. Case 1. A 62-year-old male underwent LT for multifocal HCC, combined with hepatitis $\mathrm{C}$ related cirrhosis. According to preoperative $\mathrm{CT}$ scan images, a massive lesion with greatest extent over $8 \mathrm{~cm}$ was found in the right lobe and 2-3 small lesions were found in segment 2. AFP level was elevated at $278 \mathrm{ng} / \mathrm{mL}$. Histological examination of the explanted liver revealed disseminated HCC with numerous nodules in all segments and widespread vessel infiltration. In the posttransplant period the patient received immunosuppressive therapy with tacrolimus, prednisolone, and mycophenolate mofetil (MMF).

Nine months after LT a routine CT scan revealed an extrahepatic, $2 \mathrm{~cm}$ lesion adjacent to segment 6 (Figure 1). Ultrasound guided biopsy verified HCC. The case was determined suitable for laparoscopic resection.

Pneumoperitoneum was established through an open approach via right pararectal region, supplemented by three trocars: a $12 \mathrm{~mm}$ through the scar from previous incision and two $5 \mathrm{~mm}$ in the right flank. After complete mobilization of the right lobe, the tumor was found retroperitoneally between the right kidney and adrenal gland. Intraoperative ultrasound was carried out to determine the borders of the tumor. Despite close relation with renal capsule, no signs of infiltration to the kidney were revealed. Division of Gerota's fascia and exposing the upper part of the kidney allowed mobilization of the tumor laterally and cranially, separating it from the renal capsule. No evidence of infiltration to adjacent structures was found and the tumor was removed. No significant bleeding occurred during the surgery and the operation time was $131 \mathrm{~min}$.

Histological findings confirmed the presence of two (40 $\mathrm{mm}$ and $12 \mathrm{~mm}$ ) extrahepatic recurrences from HCC. The postoperative course was uneventful.

Surveillance for the first 8 months revealed no signs of disease recurrence, but one year after the reresection, metastases in liver, lungs, and adrenal gland were detected. Subsequent therapy with sorafenib was considered as the only possible active treatment.

3.2. Case 2. HCC was diagnosed in a 57-year-old male with hepatitis $\mathrm{C}$ and cirrhosis. A $3 \mathrm{~cm}$ tumor, located in segment 5, and two small hypervascular lesions, suggestive of HCC, were discovered by CT scan. The AFP level was significantly elevated ( $>20.000)$.

The patient was assessed and accepted as a liver recipient and LT was performed. The postoperative course was uneventful and the patient received immunosuppression with tacrolimus, prednisolone, and MMF. 


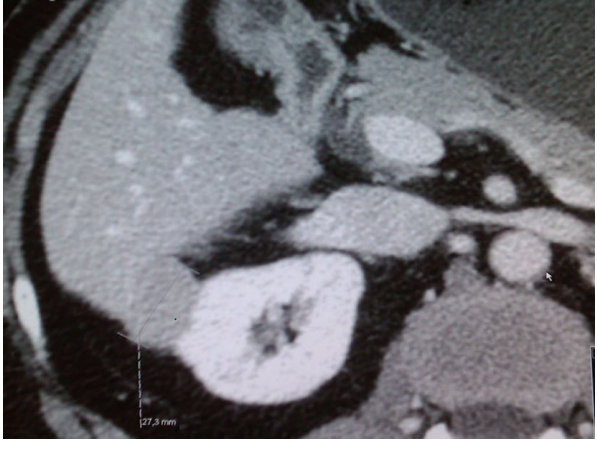

(a)

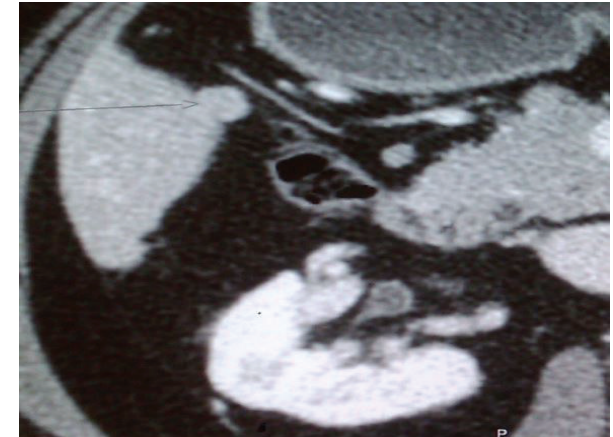

(b)

Figure 2: CT with intravenous contrast. Two lesions detected. First lesion adjacent to lateral part of right kidney, largest diameter $2.7 \mathrm{~cm}$ (a). Second lesion close to duodenum, largest diameter $1.4 \mathrm{~cm} \mathrm{(b).}$

An abdominal CT scan, 6 years after LT, detected two intrahepatic lesions. The larger one, measuring $2.7 \mathrm{~cm}$, was located laterally in segment 6 , close to the right kidney (Figure 2(a)). The second lesion of $1.4 \mathrm{~cm}$ was located between the segments 5 and 6 , adjacent to the wall of duodenum (Figure 2(b)).

The laparoscopic approach was chosen for reresection. Pneumoperitoneum was obtained through an open access technique via umbilicus. Adhesiolysis was carried out. Afterwards, one $12 \mathrm{~mm}$ trocar was placed high in the midline, followed by two $5 \mathrm{~mm}$ trocars in the right pararectal area and more laterally. Mobilizing the right lobe of the liver, the lateral tumor was found to infiltrate into Gerota's fascia. Using LigaSure (Valleylab/Covidien, Boulder, CO, USA) device, the tumor was completely mobilized and separated from the fascia. Finally, both of the tumors were removed by wedge resection of the liver. Operative time was $127 \mathrm{~min}$ with only minor intraoperative blood loss observed.

A pathological examination revealed $32 \mathrm{~mm}$ and $18 \mathrm{~mm}$ measuring moderately differentiated HCC with trabecular pattern. The postoperative course was uneventful. During further surveillance for more than 3 years, no signs of disease recurrence have been observed.

\section{Discussion}

LT is associated with significantly higher 5-year overall and disease-free survival rates, compared to LR $(65.7 \%$ versus $43.8 \% ; P=0.005$ and $85.3 \%$ versus $22.7 \% ; P<0.001$, resp.); hence it should be considered in patients with liver disease and cirrhosis within the various transplantation criteria [14, 15]. Unlike resection, LT is also an ultimate solution for treatment of HCC and underlying cirrhosis [16]. Up to 83\% 5 -year recurrence-free survival rates have been reported after LT for HCC, which is comparable to LT for nonmalignant disease [17].

Unfortunately, even the utilization of criteria for LT does not prevent tumor recurrence. According to Welker and coworkers [10], the recurrence rates following LT vary between $15 \%$ and $20 \%$. The latter was assumed to be dependent from patient selection criteria for initial LT. In another paper by Sotiropoulos and colleagues [18] a $12.6 \%$ recurrence rate was found among the patients within the Milan criteria.

Numerous retrospective analyses have been performed to estimate the factors leading to the recurrence and eventual poor outcome. Parameters such as vascular invasion, poor differentiation, tumor size $>5 \mathrm{~cm}$, number of lesions, HCC exceeding the Milan criteria, and elevated pretransplant AFP levels have been reported to have significant impact on the rate of posttransplant recurrences [18-20].

The limitations of therapeutic options for recurrent HCC after LT lead to an absence of established treatment strategies $[18,21]$. A major contraindication for reresection is the presence of extrahepatic recurrences that have been described in $10-43 \%$ of patients. These are typically located in lungs, bones, abdominal lymph nodes, and adrenal glands [22]. Nevertheless, even though disseminated disease commonly precludes curative treatment, patients with limited intraor extrahepatic lesions may still benefit from local treatment, including surgical resection [17]. Roayaie et al. [12] reported that recurrent disease should be treated by surgery whenever possible, since it was independently associated with prolonged survival of $47 \%$ at 5 years. Hwang et al. [23] recommended performing pulmonary metastasectomy for resectable HCC metastases in lungs following LT, as it may provide a chance of long-term survival in a substantial proportion of patients. According to recent clinical studies, resection of both intra- and/or extrahepatic lesions in a selected group of patients can be associated with an improved overall 5-year survival ranging from 27 to $88 \%$ [22, 24-26]. Furthermore, the authors conclude that even noncurative surgery seems to prolong survival [18]. Reportedly, resection rates for recurrent HCC may vary from 31 to $43 \%$ among different centers [19]. However, the studies are limited by retrospective design and a small series of cases, reported so far (Table 1).

Another available surgical option seems to be retransplantation. Nevertheless, the attempts are currently made to salvage graft rather than performing retransplantation, because of the organ shortage. Hence, although the resections are relatively rare, they are associated with favorable outcome 
TABLE 1: Recurrence and resection rates following liver transplantation.

\begin{tabular}{lcccc}
\hline Authors & Year of publication & Liver transplantations & Total number of recurrences & Resections for recurrent HCC \\
\hline Pfeiffenberger et al. [29] & 2013 & 136 & 18 & $3(16.6 \%)$ \\
Hwang et al. [23] & 2012 & 587 & 43 & $23^{*}$ \\
Chok et al. [16] & 2011 & 139 & 24 & $17(70.8 \%)$ \\
Pfiffer et al. [24] & 2011 & 139 & 24 & $7(29.1 \%)$ \\
Kornberg et al. [17] & 2010 & 60 & 16 & $7(44 \%)$ \\
Shin et al. [30] & 2010 & 138 & 28 & $4(14.3 \%)$ \\
Valdivieso et al. [19] & 2010 & 182 & 23 & $11(47.8 \%)$ \\
Taketomi et al. [25] & 2010 & 101 & 17 & $9(52.9 \%)$ \\
Roayaie et al. [12] & 2004 & 311 & 57 & $15(32 \%)$ \\
Schlitt et al. [11] & 1999 & 69 & 39 & $15(38 \%)$ \\
Regalia et al. [26] & 1998 & 132 & 21 & $7(32 \%)$ \\
\hline
\end{tabular}

${ }^{*}$ Hwang et al. reported on 43 HCC cases with pulmonary recurrences after LT. 23 patients underwent resections.

in selected patients and avoid retransplantation in most of the cases [27].

There has been a considerable development in laparoscopic liver surgery during the last decade and it is now generally accepted that laparoscopic resection offers advantages, compared to the open approach in terms of severity of complications, 30-day readmission rate, length of hospital stay, and blood loss [28]. However, no reports in the literature concerning laparoscopic resections for recurrent HCC after LT have been observed to date.

In this report we present two cases that may suggest that laparoscopic resection is a feasible and safe procedure in selected patients with recurrent HCC following LT. Since these patients often have a dismal prognosis, emphasis should be on minimizing surgical risk and trauma when choosing therapeutic options for treatment of recurrence. In this context laparoscopy seems to be a rational approach that may be well suited in selected patients. The disparity in longterm outcome in two patients can probably be related to the difference between the risk factors for recurrence. While the first patient exceeded all morphological criteria systems, the second patient fulfilled each of them (BCLC, Milan, UGSF, and "up-to-seven"). On the other hand, the occurrence of socalled "de novo" cancer cannot be excluded in Case 2, considering the fact that the lesions developed 6 years after LT.

In the light of the limited role of medical therapy in palliative treatment of recurrent HCC, patients with poor prognosis and limited survival prospects can benefit from laparoscopy due to shortened recovery period. In case of indications for subsequent therapy, laparoscopy may avoid potential delay in treatment, caused by open surgery. Conversely, the patients with favorable prognosis may also benefit from the fast recovery course, provided by laparoscopy. Extensive experience in the field of laparoscopic surgery coupled with the evaluation of therapeutic options will establish the new surgical guidelines in treatment of resectable recurrent HCC after LT.

\section{Conflict of Interests}

The authors declare that there is no conflict of interests regarding the publication of this paper.

\section{References}

[1] S. Bhoori and V. Mazzaferro, "Current challenges in liver transplantation for hepatocellular carcinoma," Best Practice \& Research Clinical Gastroenterology, vol. 28, no. 5, pp. 867-879, 2014.

[2] F. Pons, M. Varela, and J. M. Llovet, "Staging systems in hepatocellular carcinoma," HPB, vol. 7, no. 1, pp. 35-41, 2005.

[3] P.-A. Clavien, M. Lesurtel, P. M. Bossuyt, G. J. Gores, B. Langer, and A. Perrier, "Recommendations for liver transplantation for hepatocellular carcinoma: an international consensus conference report," The Lancet Oncology, vol. 13, no. 1, pp. ell-e22, 2012.

[4] V. Mazzaferro, E. Regalia, R. Doci et al., "Liver transplantation for the treatment of small hepatocellular carcinomas in patients with cirrhosis," The New England Journal of Medicine, vol. 334, no. 11, pp. 693-699, 1996.

[5] F. Y. Yao, L. Ferrell, N. M. Bass et al., "Liver transplantation for hepatocellular carcinoma: expansion of the tumor size limits does not adversely impact survival," Hepatology, vol. 33, no. 6, pp. 1394-1403, 2001.

[6] V. Mazzaferro, J. M. Llovet, R. Miceli et al., "Predicting survival after liver transplantation in patients with hepatocellular carcinoma beyond the Milan criteria: a retrospective, exploratory analysis," The Lancet Oncology, vol. 10, no. 1, pp. 35-43, 2009.

[7] D. A. Kooby, V. Egnatashvili, M. Graiser et al., "Changing management and outcome of hepatocellular carcinoma: evaluation of 501 patients treated at a single comprehensive center," Journal of Surgical Oncology, vol. 98, no. 2, pp. 81-88, 2008.

[8] K. V. Menon, A. R. Hakeem, and N. D. Heaton, "Review article: liver transplantation for hepatocellular carcinoma-a critical appraisal of the current worldwide listing criteria," Alimentary Pharmacology and Therapeutics, vol. 40, no. 8, pp. 893-902, 2014.

[9] J.-O. Lee, D.-Y. Kim, J. H. Lim et al., "Palliative chemotherapy for patients with recurrent hepatocellular carcinoma after liver transplantation," Journal of Gastroenterology and Hepatology, vol. 24, no. 5, pp. 800-805, 2009.

[10] M.-W. Welker, W.-O. Bechstein, S. Zeuzem, and J. Trojan, "Recurrent hepatocellular carcinoma after liver transplantation-an emerging clinical challenge," Transplant International, vol. 26, no. 2, pp. 109-118, 2013. 
[11] H. J. Schlitt, M. Neipp, A. Weimann et al., "Recurrence patterns of hepatocellular and fibrolamellar carcinoma after liver transplantation," Journal of Clinical Oncology, vol. 17, no. 1, pp. 324331, 1999.

[12] S. Roayaie, J. D. Schwartz, M. W. Sung et al., "Recurrence of hepatocellular carcinoma after liver transplant: patterns and prognosis," Liver Transplantation, vol. 10, no. 4, pp. 534-540, 2004.

[13] T. Kamiyama, M. Tahara, K. Nakanishi et al., "Long-term outcome of laparoscopic hepatectomy in patients with hepatocellular carcinoma," Hepatogastroenterology, vol. 61, no. 130, pp. 405-409, 2014.

[14] M. H. Squires III, S. I. Hanish, S. B. Fisher et al., "Transplant versus resection for the management of hepatocellular carcinoma meeting Milan Criteria in the MELD exception era at a single institution in a UNOS region with short wait times," Journal of Surgical Oncology, vol. 109, no. 6, pp. 533-541, 2014.

[15] R. J. Wong, J. Wantuck, A. Valenzuela et al., "Primary surgical resection versus liver transplantation for transplant-eligible hepatocellular carcinoma patients," Digestive Diseases and Sciences, vol. 59, no. 1, pp. 183-191, 2014.

[16] K. S. H. Chok, S. C. Chan, T. T. Cheung, A. C. Y. Chan, S. T. Fan, and C. M. Lo, "Late recurrence of hepatocellular carcinoma after liver transplantation," World Journal of Surgery, vol. 35, no. 9, pp. 2058-2062, 2011.

[17] A. Kornberg, B. Küpper, A. Tannapfel et al., "Long-term survival after recurrent hepatocellular carcinoma in liver transplant patients: clinical patterns and outcome variables," European Journal of Surgical Oncology, vol. 36, no. 3, pp. 275-280, 2010.

[18] G. C. Sotiropoulos, E. P. Molmenti, C. Lösch, S. Beckebaum, C. E. Broelsch, and H. Lang, "Meta-analysis of tumor recurrence after liver transplantation for hepatocellular carcinoma based on 1,198 cases," European Journal of Medical Research, vol. 12, no. 10, pp. 527-534, 2007.

[19] A. Valdivieso, J. Bustamante, M. Gastaca et al., "Management of hepatocellular carcinoma recurrence after liver transplantation," Transplantation Proceedings, vol. 42, no. 2, pp. 660-662, 2010.

[20] M. Abdel-Wahab, A. M. Sultan, O. M. Fathy et al., "Factors affecting recurrence and survival after living donor liver transplantation for hepatocellular carcinoma," HepatoGastroenterology, vol. 60, no. 128, pp. 1847-1853, 2013.

[21] H. R. Kim, S. H. Cheon, S. Y. Rha et al., "Treatment of recurrent hepatocellular carcinoma after liver transplantation," Asia-Pacific Journal of Clinical Oncology, vol. 7, no. 3, pp. 258269, 2011.

[22] P. J. Kneuertz, D. P. Cosgrove, A. M. Cameron et al., "Multidisciplinary management of recurrent hepatocellular carcinoma following liver transplantation," Journal of Gastrointestinal Surgery, vol. 16, no. 4, pp. 874-881, 2012.

[23] S. Hwang, Y.-H. Kim, D. K. Kim et al., "Resection of pulmonary metastases from hepatocellular carcinoma following liver transplantation," World Journal of Surgery, vol. 36, no. 7, pp. 15921602, 2012.

[24] T. E. F. Pfiffer, D. Seehofer, A. Nicolaou, R. Neuhaus, H. Riess, and R. U. Trappe, "Recurrent hepatocellular carcinoma in liver transplant recipients: parameters affecting time to recurrence, treatment options and survival in the sorafenib era," Tumori, vol. 97, no. 4, pp. 436-441, 2011.

[25] A. Taketomi, T. Fukuhara, K. Morita et al., "Improved results of a surgical resection for the recurrence of hepatocellular carcinoma after living donor liver transplantation," Annals of Surgical Oncology, vol. 17, no. 9, pp. 2283-2289, 2010.

[26] E. Regalia, L. R. Fassati, U. Valente et al., "Pattern and management of recurrent hepatocellular carcinoma after liver transplantation," Journal of Hepato-Biliary-Pancreatic Surgery, vol. 5, no. 1, pp. 29-34, 1998.

[27] G. Marangoni, W. Faraj, H. Sethi, M. Rela, P. Muiesan, and N. Heaton, "Liver resection in liver transplant recipients," Hepatobiliary and Pancreatic Diseases International, vol. 7, no. 6, pp. 590-594, 2008.

[28] J. J. Lee, J. B. Conneely, R. L. Smoot et al., "Laparoscopic versus open liver resection for hepatocellular carcinoma at a NorthAmerican Centre: a 2-to-1 matched pair analysis," $H P B$, vol. 17, no. 4, pp. 304-310, 2015.

[29] J. Pfeiffenberger, R. Koschny, K. Hoffmann et al., "Sorafenib treatment is save and may affect survival of recurrent hepatocellular carcinoma after liver transplantation," Langenbeck's Archives of Surgery, vol. 398, no. 8, pp. 1123-1128, 2013.

[30] W. Y. Shin, K.-S. Suh, H. W. Lee et al., "Prognostic factors affecting survival after recurrence in adult living donor liver transplantation for hepatocellular carcinoma," Liver Transplantation, vol. 16, no. 5, pp. 678-684, 2010. 


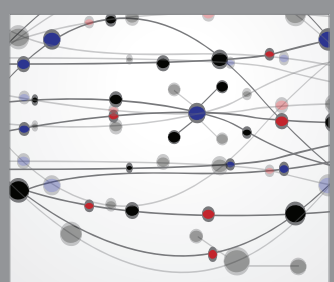

The Scientific World Journal
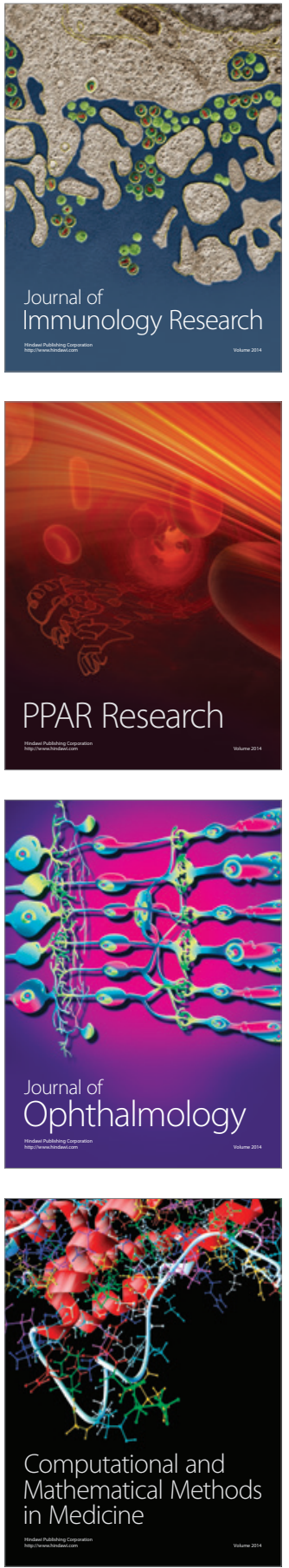

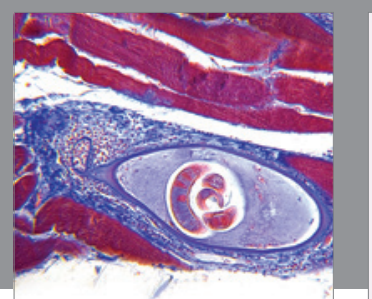

Gastroenterology Research and Practice

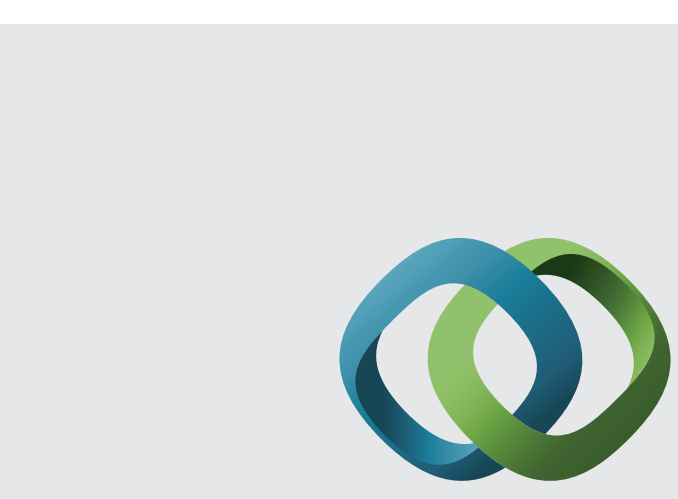

\section{Hindawi}

Submit your manuscripts at

http://www.hindawi.com
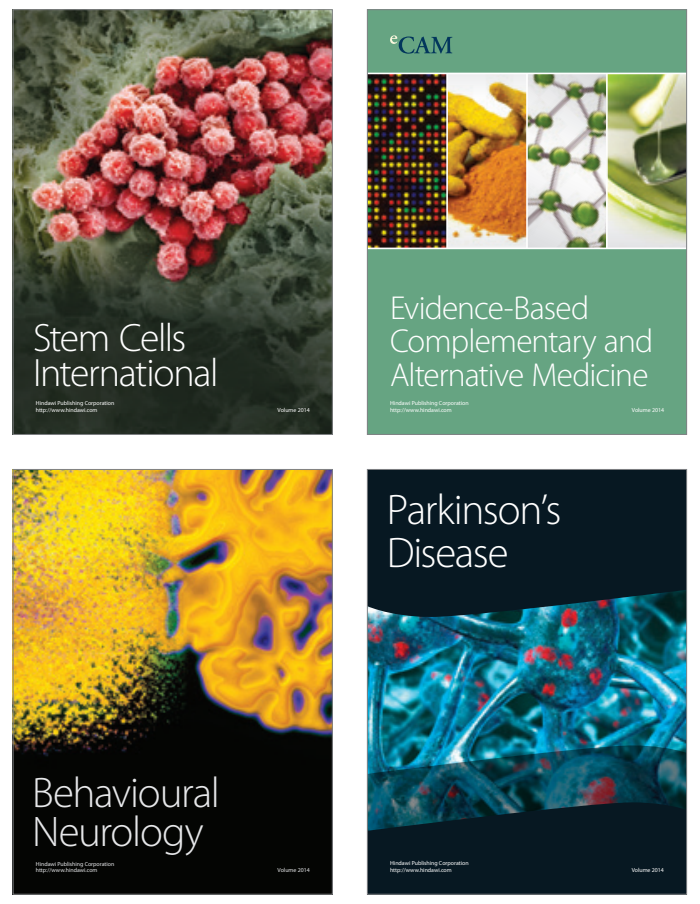
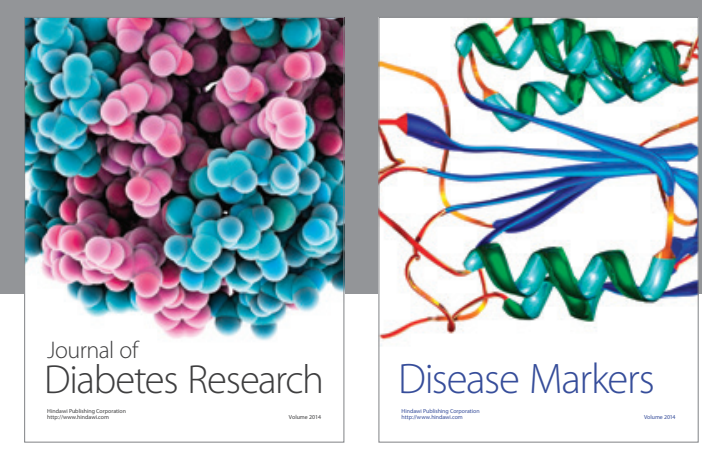

Disease Markers
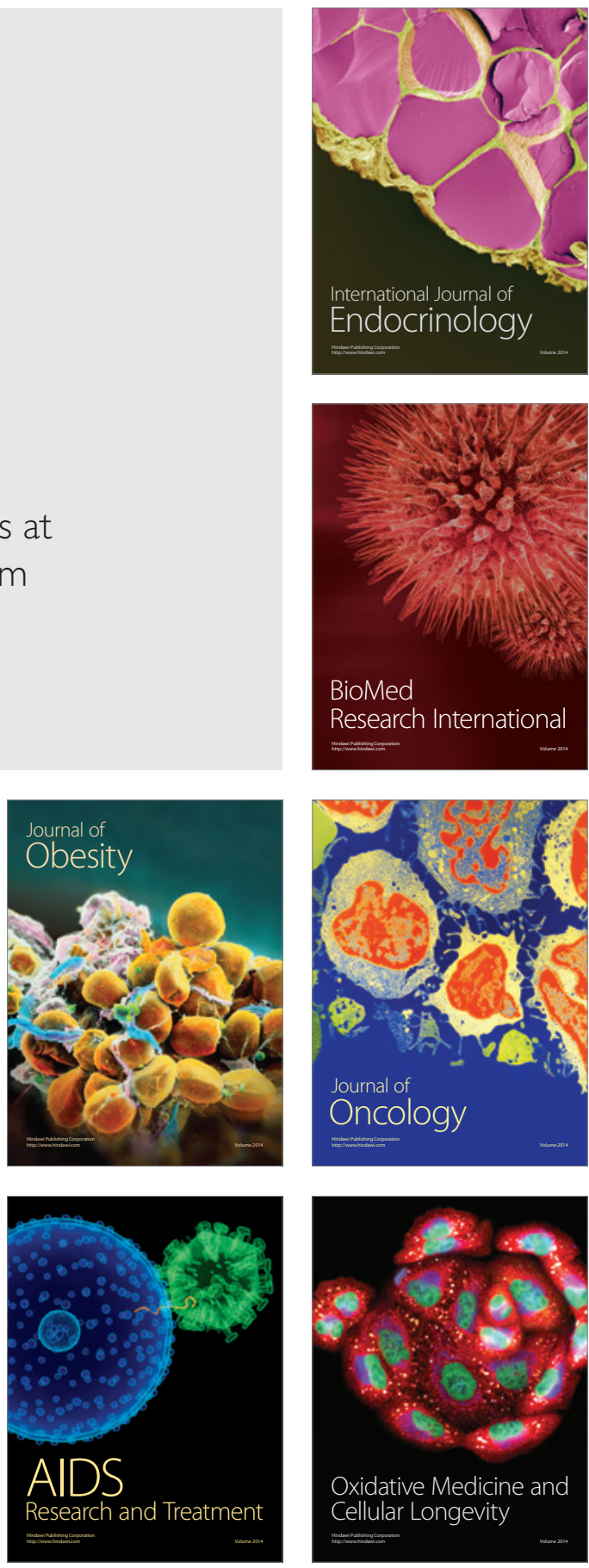